\title{
Factors Affecting the Development of Metabolic Syndrome in Single-Person Households: A Sex-Stratified Analysis
}

\author{
Jui Kim ${ }^{1}$ and Hyoungshim Choi ${ }^{2, *}$ (D) \\ 1 Department of Nursing, Ansan University, Ansan 15328, Korea; juikim@ansan.ac.kr \\ 2 Department of Nursing, Hansei University, Gunpo 15852, Korea \\ * Correspondence: hyoungshim@hansei.ac.kr; Tel.: +82-031-450-5308
}

check for updates

Citation: Kim, J.; Choi, H. Factors Affecting the Development of Metabolic Syndrome in Single-Person Households: A Sex-Stratified Analysis. Sustainability 2021, 13, 9032. https://doi.org/10.3390/su13169032

Academic Editors: Valeria Bellisario and Giulia Squillacioti

Received: 8 July 2021

Accepted: 9 August 2021

Published: 12 August 2021

Publisher's Note: MDPI stays neutral with regard to jurisdictional claims in published maps and institutional affiliations.

Copyright: (c) 2021 by the authors. Licensee MDPI, Basel, Switzerland. This article is an open access article distributed under the terms and conditions of the Creative Commons Attribution (CC BY) license (https:// creativecommons.org/licenses/by/ $4.0 /)$.

\begin{abstract}
Various sociodemographic and behavioral health factors have been reported to influence the development of metabolic syndrome (Mets). In addition, the effects of various sociodemographic and health related characteristics on single-person households appear to be influenced by sex. Considering the surge in the number of single-person households globally, we conducted a sexstratified analysis of the factors that affect the development of Mets among single-person Korean households. We used secondary data from the 2016-2018 Korea National Health and Nutrition Examination Survey (KNHANES) for this study. A total of 1830 participants (aged $\geq 19$ years) without any missing data were analyzed in this study. The chi-square test and multiple logistic regression were used to analyze the study data. The results of the multiple logistic regression analysis showed that the significant risk factors for Mets in men were age, body mass index (BMI), subjective health status, subjective recognition of body shape, household income, and sleep hours, whereas age, BMI, subjective health status, exercise, and sleep hours were the significant risk factors for Mets in women. Specialized sex-specific health policies and programs are needed to reduce the prevalence of Mets in single-person households.
\end{abstract}

Keywords: metabolic syndrome; single person; diagnostic self-evaluation; body mass index; nutrition surveys

\section{Introduction}

The surge in the number of single-person households is a global phenomenon [1]. The proportion of single-person households in Europe was 34\% as of 2017, 28\% in the United States as of 2018, and 35\% in Japan as of 2015 [2-4]. In South Korea, the proportion of single-person households was $28.5 \%$ as of 2019 and is expected to increase rapidly to $35.7 \%$ in 2037 [5,6]. The interest in single-person households is increasing because single-person households are generally weaker in terms of housing, safety, health, income, and social networks than multi-person households. Thus, an increase in the number of single-person households leads to an increase in the instability of society as a whole [7]. According to previous studies, the income levels, education levels, and quality of life of single-person households are lower than those of multi-family households [7-9]. In addition, the drinking rate, smoking rate, and prevalence of metabolic syndrome (Mets) and chronic diseases, which are also known as lifestyle diseases, are higher in single-person households than in multi-family households [7-9].

Mets is a collection of risk factors, including high blood pressure, increased fasting blood sugar level, dyslipidemia (high triglyceride and low high-density lipoprotein [HDL] levels), and abdominal obesity, that increase the risk of heart disease, diabetes, and stroke [10]. Mets is a major global public health challenge because it increases the risk of death caused by cardiovascular disease and diabetes [11]. The prevalence of Mets varies from country to country: the prevalence is 34.7\% in the United States (2011-2012), 24.2\% in China (2010-2012), and 24.0\% in South Korea (2017). These can all be regarded as high 
values [12-14]. The prevalence of Mets is expected to increase in the future due to the increased consumption of meat-based foods, sedentary lifestyles, stress, lack of exercise, and the aging of the population [15]. The prevalence of Mets in single households (26.4\%) was significantly higher $\left(\chi^{2}=7.81, p=0.017\right)$ than in multi-family households $(19.5 \%)$ of 4939 South Korean adults aged 20-64 years old from the 2013-2015 Korea National Health and Nutrition Examination Survey (KNHANES) [9].

Various demographic factors, such as sex, age, income, education level, employment, household type, and behavioral health factors, such as exercise, sleep, smoking, drinking, and regular health check-ups are known to influence the development of Mets $[8,14,16]$. Socioeconomic factors, including old age, low income, low education level, unemployment, and single-person households, are also known to increase the risk of Mets $[8,9,13,17]$. The sex-specific prevalence rate of Mets differs according to country, age, and race. The prevalence of Mets in adults differs from country to country as follows: $32.8 \%$ for men and $36.6 \%$ for women in the United States (2011-2012), $24.6 \%$ for men and $23.8 \%$ for women in China (2010-2012), and 28.1\% for men and 18.7\% for women in South Korea (2017) [12-14]. Factors affecting the development of Mets also vary according to sex. Women who experience occupational stress, have low socioeconomic conditions, such as low income and low education levels, and do not exercise frequently are more likely to develop Mets than men with the same conditions $[15,18]$. On the other hand, the severity of drinking and smoking has been reported to be an important factor for the development of Mets in men [15,18].

The effects of various health behaviors on persons from single-person households appear to be influenced by sex, age, income level, and education level. In models that included socio-demographics such as income and education level, the significant health behavioral risk factors of Mets of Korean single-person households varied [8,9]. The severity of drinking and smoking was a common significant factor, but the impact of exercise was controversial [8,9]. Therefore, it is necessary to evaluate factors affecting the development of Mets in single-person households by assessing the demographic and sociological characteristics of the single-person household population $[8,15]$. The factors that influence the development of Mets have been separately analyzed in previous studies according to sex and household type. However, only a few studies have involved the sexspecific analysis of these factors among subjects from single-person households. Therefore, this study aimed to provide basic data for the development of interventions tailored to the sex-specific characteristics of persons from single-person households by conducting a sexstratified evaluation of the factors that influence the development of Mets in single-person households using representative national data.

\section{Materials and Methods}

\subsection{Study Design and Participants}

This cross-sectional study was conducted using the data extracted from the 2016-2018 KNHANES. The KNHANES is a national representative survey conducted by the Korean Ministry of Health and Welfare.

We recruited 19,389 participants aged 19 years or older who participated in the health examination survey in the KNHANES from 2016 to 2018. We excluded multi-person households $(n=17,067)$ and persons with missing data $(n=492)$. A total of 1830 participants were included in the final analyses.

\subsection{Sociodemographic and Health-Related Characteristics}

Data on sociodemographic and health-related characteristics were obtained from self-report questionnaires and personal interviews conducted by trained staff. The sociodemographic characteristics included sex, age (younger than 50 years old, over 50 years and under 65 years, or 65 years or older), subjective health status (good, normal, or poor), subjective recognition of body shape (thin, normal, or fat), level of education (graduated from a college or higher or graduated from a high school or less), household income (low, 
mid-low, mid-high, or high), employment (yes or no), and food security (enough or not enough). The health-related characteristics considered were alcohol intake (drinks more than twice a month or drinks less than twice a month), smoking status (smoked more than 100 cigarettes in their lifetime or smoked less than 100 cigarettes in their lifetime), exercise (moderate-intensity physical activity or more, or less than moderate-intensity physical activity), and sleep hours ( $8 \mathrm{~h}$ or longer, or less than $8 \mathrm{~h}$ ).

\subsection{Anthropometric and Laboratory Measurements}

Height and body weight were measured by the health staff using a standardized protocol. Body mass index (BMI) was calculated as weight $(\mathrm{kg}) /$ height $\left(\mathrm{m}^{2}\right)$ and categorized into underweight $\left(<18.5 \mathrm{~kg} / \mathrm{m}^{2}\right)$, normal to pre-obese $\left(18.5-24.9 \mathrm{~kg} / \mathrm{m}^{2}\right)$, and obese class I-III ( $\geq 25 \mathrm{~kg} / \mathrm{m}^{2}$ ) according to the Asia-Pacific obesity guidelines [19]. Waist circumference (WC) was measured at a point midway between the tenth rib and the iliac crest and recorded to the nearest centimeter [20]. Blood pressure was measured by a skilled nurse. Mets was defined according to the criteria outlined by the National Cholesterol Education Program Adult Treatment Panel III [21], which was modified for the Asian population. The presence of three or more of the following criteria constituted a diagnosis of Mets: (1) WC $\geq 85 \mathrm{~cm}$; (2) systolic BP $\geq 130 \mathrm{mmHg}$ or diastolic BP $\geq 85 \mathrm{mmHg}$; (3) fasting plasma glucose $\geq 100 \mathrm{mg} / \mathrm{d}$; (4) triglyceride level $\geq 150 \mathrm{mg} / \mathrm{dL}$; and (5) HDL-cholesterol level $<50 \mathrm{mg} / \mathrm{dL}$.

\subsection{Statistical Analysis}

SPSS version 26 (SPSS Inc., Chicago, IL, USA) was used to analyze the study data. A $p$-value $<0.05$ was considered statistically significant. The chi-square test was used to analyze the general characteristics, socioeconomic status, health behaviors, and Mets components of the study participants according to sex. The difference in sex-stratified Mets prevalence according to sociodemographic and health-related characteristics was also compared using the chi-square test. We applied linear trend tests for ordinal variables. Multiple logistic regression was used for the assessment of variables associated with Mets according to sex strata.

\section{Results}

The sociodemographic data, health-related characteristics, and Mets components of the study participants are outlined in Table 1. Of the 1830 participants included in this study, 714 were men, and 1116 were women. Most of the male subjects in this study were under 50 years (42.9\%). The men in this study showed high proportions of a BMI of 18.5-24.9 (57.3\%), a subjective health status rated as "normal" (49.9\%), a subjective body shape recognition as "normal" (43.4\%), graduated from a high school or less (65.1\%), low household income (38.4\%), employed (60.5\%), felt enough food security (90.2\%), consumed alcohol (61.9\%), current smoking status $(76.8 \%)$, did not exercise $(71.8 \%)$, slept for over $8 \mathrm{~h}(71.4 \%)$, and no Mets $(70.7 \%)$. Most female subjects in this study were aged over 65 years $(59.8 \%)$. The women in this study showed high proportions of a BMI of 18.5-24.9 (53.0\%), a subjective health status rated as "normal" (49.3\%), a subjective body shape recognition as "fat" (44.0\%), graduated from a high school or less $(84.1 \%)$, low household income (59.1\%), not employed (56.1\%), felt enough food security (92.7\%), no alcohol consumption $(76.1 \%)$, no smoking status $(87.5 \%)$, did not exercise $(85.4 \%)$, slept for over $8 \mathrm{~h}(68.0 \%)$, and no Mets $(64.0 \%)$. There were significant differences in age $(p<0.0001)$, subjective health status $(p<0.0001)$, subjective recognition of body shape $(p<0.0001)$, level of education $(p<0.0001)$, household income $(p<0.0001)$, employment $(p<0.0001)$, alcohol intake $(p<0.0001)$, smoking status $(p<0.0001)$, exercise $(p<0.0001)$, abdominal obesity $(p<0.0001)$, triglyceride level $(p<0.0001)$, HDL cholesterol level $(p<0.0001)$, and metabolic syndrome $(p=0.003)$ between men and women. 
Table 1. Sociodemographic and health-related characteristics and metabolic syndrome components of the study participants $(N=1830)$.

\begin{tabular}{|c|c|c|c|c|c|}
\hline Variable & Categories & $\begin{array}{c}\text { Men } \\
(N=714) N(\%)\end{array}$ & $\begin{array}{c}\text { Women } \\
(N=1116) N(\%)\end{array}$ & $x^{2}$ & $\begin{array}{c}\text { Linear } \\
\text { Trend Test }\end{array}$ \\
\hline \multicolumn{6}{|l|}{ Sociodemographic characteristics } \\
\hline \multirow{3}{*}{ Age } & $<50$ & $306(42.9)$ & $218(19.5)$ & $162.971^{* * *}$ & $161.605^{* * *}$ \\
\hline & $50-64$ & $187(26.2)$ & $231(20.7)$ & & \\
\hline & $\geq 65$ & $221(31.0)$ & $667(59.8)$ & & \\
\hline \multirow{3}{*}{ BMI, $\mathrm{kg} / \mathrm{m}^{2}$} & $<18.5$ & $28(3.9)$ & $43(3.9)$ & 3.524 & 2.776 \\
\hline & $18.5 \sim 24.9$ & $409(57.3)$ & $591(53.0)$ & & \\
\hline & $\geq 25$ & $277(38.8)$ & $482(43.2)$ & & \\
\hline \multirow{3}{*}{ Subjective health status } & Good & $190(26.6)$ & $169(15.1)$ & $49.674^{* * *}$ & $48.870 * * *$ \\
\hline & normal & $356(49.9)$ & $550(49.3)$ & & \\
\hline & Poor & $168(23.5)$ & $397(35.6)$ & & \\
\hline \multirow{3}{*}{ Subjective body shape recognition } & Thin & $161(22.5)$ & $181(16.2)$ & $21.506^{* * *}$ & $21.311^{* * *}$ \\
\hline & normal & $310(43.4)$ & $444(39.8)$ & & \\
\hline & Fat & $243(34.0)$ & $491(44.0)$ & & \\
\hline \multirow{2}{*}{ Level of education } & $\begin{array}{l}\text { Graduated from a } \\
\text { college or higher }\end{array}$ & $249(34.9)$ & $177(15.9)$ & $88.140^{* * *}$ & $88.092^{* * *}$ \\
\hline & $\begin{array}{l}\text { Graduated from a } \\
\text { high school or less }\end{array}$ & $465(65.1)$ & $939(84.1)$ & & \\
\hline \multirow{4}{*}{ Household income } & Low & $274(38.4)$ & $660(59.1)$ & $112.507^{* * *}$ & $112.036^{* * *}$ \\
\hline & Mid-low & $167(23.4)$ & $251(22.5)$ & & \\
\hline & Mid-high & $129(18.1)$ & $126(11.3)$ & & \\
\hline & High & $144(20.2)$ & $79(7.1)$ & & \\
\hline \multirow{2}{*}{ Employment } & Yes & $432(60.5)$ & $490(43.9)$ & $47.982^{* * *}$ & \\
\hline & No & $282(39.5)$ & $626(56.1)$ & & \\
\hline \multirow{2}{*}{ Food security } & Enough & $644(90.2)$ & $1034(92.7)$ & 3.449 & 3.447 \\
\hline & Not enough & $70(9.8)$ & $82(7.3)$ & & \\
\hline \multicolumn{6}{|l|}{ Health-related characteristics } \\
\hline \multirow{2}{*}{ Alcohol intake } & No & $272(38.1)$ & $849(76.1)$ & $264.650 * * *$ & \\
\hline & Yes & $442(61.9)$ & $267(23.9)$ & & \\
\hline \multirow{2}{*}{ Smoking status } & No & $166(23.2)$ & $976(87.5)$ & $765.083^{* * *}$ & \\
\hline & Yes & $548(76.8)$ & $140(12.5)$ & & \\
\hline \multirow{2}{*}{ Exercise } & No & $513(71.8)$ & $953(85.4)$ & $50.138^{* * *}$ & \\
\hline & Yes & $201(28.2)$ & $163(14.6)$ & & \\
\hline \multirow{2}{*}{ Sleep hours } & $\geq 8$ & $510(71.4)$ & $759(68.0)$ & 2.393 & 2.391 \\
\hline & $<8$ & $204(28.6)$ & $357(32.0)$ & & \\
\hline \multicolumn{6}{|l|}{ Metabolic syndrome components } \\
\hline \multirow{2}{*}{ Abdominal obesity } & Yes & $217(30.4)$ & $462(41.4)$ & $22.600 * * *$ & \\
\hline & No & 497 (69.6) & $654(58.6)$ & & \\
\hline \multirow{2}{*}{ Hypertension } & Yes & $287(40.2)$ & $451(40.4)$ & 0.008 & \\
\hline & No & $427(59.8)$ & $665(59.6)$ & & \\
\hline \multirow{2}{*}{ Hyperglycemia } & Yes & $322(45.1)$ & $483(43.3)$ & 0.584 & \\
\hline & No & $392(54.9)$ & $633(56.7)$ & & \\
\hline \multirow{2}{*}{ High Triglyceride } & Yes & $262(36.7)$ & $296(26.5)$ & $21.255^{* * *}$ & \\
\hline & No & $452(63.3)$ & $820(73.5)$ & & \\
\hline \multirow{2}{*}{ Low HDL cholesterol } & Yes & $196(27.5)$ & $522(46.8)$ & $68.193^{* * *}$ & \\
\hline & No & $518(72.5)$ & $594(53.2)$ & & \\
\hline \multirow{2}{*}{ Metabolic syndrome } & Yes & $209(29.3)$ & $402(36.0)$ & $8.920^{* *}$ & \\
\hline & No & 505 (70.7) & $714(64.0)$ & & \\
\hline
\end{tabular}


Table 2 shows the differences in the prevalence of Mets among men and women according to sociodemographic and health-related characteristics. The men with Mets in this study were mostly aged 65 years or older $(35.9 \%)$, had a BMI over $25(69.4 \%)$, a perceived subjective health status as "normal" (52.2\%) and a subjective body shape recognition as "fat" (56.5\%), graduated from a high school or less $(68.9 \%)$, had a low household income $(44.5 \%)$, were employed (58.4\%), felt enough food security $(85.6 \%)$, consumed alcohol (59.3\%), had a current smoking status (79.4\%), did not exercise (78.5\%), and slept over $8 \mathrm{~h}(64.6 \%)$. The women with Mets in this study were mostly aged 65 years or older $(76.1 \%)$, had a BMI of $18.5-24.9(29.9 \%)$, a perceived subjective health status as "normal" (47.3\%) and a subjective body shape recognition as "fat" (57.5\%), graduated from a high school or less (93.8\%), had a low household income $(72.1 \%)$, were unemployed $(67.9 \%)$, felt enough food security $(91.0 \%)$, did not consume alcohol $(83.8 \%)$, did not have current smoking status $(88.6 \%)$, did not exercise $(92.3 \%)$, and slept for over $8 \mathrm{~h}(65.2 \%)$. There were significant differences in age $(p=0.002)$, BMI $(p<0.0001)$, subjective health status $(p<0.0001)$, subjective body shape recognition $(p<0.0001)$, household income $(p=0.016)$, food security $(p=0.009)$, exercise $(p=0.011)$, and sleep hours $(p=0.009)$ between the men who did and did not have Mets, whereas age $(p<0.0001)$, BMI $(p<0.0001)$, subjective health status $(p<0.0001)$, subjective body shape recognition $(p<0.0001)$, level of education $(p<0.0001)$, household income ( $\mathrm{p}<0.0001)$, employment $(p<0.0001)$, alcohol intake $(p<0.0001)$, and exercise $(p<0.0001)$ were significantly different between the women who did and did not have Mets.

Table 3 shows the results of the multiple logistic regression analysis conducted to identify the factors that significantly affect the prevalence of Mets according to sex. We used a logistic regression analysis by including variables with significant differences in the prevalence of Mets in the chi-square test (age, BMI, subjective health status, subjective body shape recognition, level of education, household income, employment, food security, alcohol intake, exercise, and sleep hours). For men, the prevalence of Mets was higher for those aged 50-64 years old (odds ratio $[\mathrm{OR}]=1.996$, confidence interval $[\mathrm{CI}]=1.199-3.322$ ) and $\geq 65$ years old $(\mathrm{OR}=1.846, \mathrm{CI}=1.063-3.204)$ than for those aged $<50$ years old. The prevalence of Mets was higher for men with a $\mathrm{BMI} \geq 25(\mathrm{OR}=7.963, \mathrm{CI}=1.587-39.949)$ than for those with a BMI < 18.5. Men with a normal subjective health status $(\mathrm{OR}=1.805$, $\mathrm{CI}=1.106-2.946)$ and a poor subjective health status $(\mathrm{OR}=2.070, \mathrm{CI}=1.169-3.667)$ were more likely to have Mets than those with a good subjective health status. Men who subjectively recognized their body shape as "normal" (OR $=2.930, \mathrm{CI}=1.466-5.857$ ) and "fat" (OR $=4.674, \mathrm{CI}=2.082-10.495)$ were more likely to have Mets than those who subjectively recognized their body shape as "thin". The prevalence of Mets was lower in men with a mid-high household income $(\mathrm{OR}=0.429, \mathrm{CI}=0.217-850)$ than in those with a low household income. The prevalence of Mets was higher in men who slept for $<8 \mathrm{~h}$ $(\mathrm{OR}=1.782, \mathrm{CI}=1.180-2.690)$ than in those who slept for $\geq 8 \mathrm{~h}$.

For women, the prevalence of Mets was higher in those aged 50-64 years old (OR $=5.568$, $\mathrm{CI}=2.924-10.606)$ and $\geq 65$ years old $(\mathrm{OR}=6.001, \mathrm{CI}=3.088-11.661)$ than in those aged $<50$ years old. The prevalence of Mets was higher in participants with a BMI 18.5-24.9 $(\mathrm{OR}=8.176, \mathrm{CI}=1.077-62.045)$ and $\geq 25(\mathrm{OR}=34.636, \mathrm{CI}=4.461-268.934)$ than in those with $\mathrm{BMI}<18.5$. Participants with a poor subjective health status $(\mathrm{OR}=1.819, \mathrm{CI}=1.111-2.980)$ were more likely to have Mets than those with a good subjective health status. The prevalence of Mets was lower in women who exercised $(\mathrm{OR}=0.568, \mathrm{CI}=0.350-0.921)$ than in those who did not exercise. The prevalence of Mets was higher in women who slept for $<8 \mathrm{~h}(\mathrm{OR}=1.392, \mathrm{CI}=1.024-1.893)$ than in those who slept for $\geq 8 \mathrm{~h}$. 
Table 2. Differences in the prevalence of metabolic syndrome according to sociodemographic and health-related characteristics $(N=1830)$.

\begin{tabular}{|c|c|c|c|c|c|c|c|c|c|}
\hline \multirow[b]{2}{*}{ Variable } & \multirow[b]{2}{*}{ Categories } & \multicolumn{4}{|c|}{ Men $(N=714)$} & \multicolumn{4}{|c|}{ Women $(N=1116)$} \\
\hline & & $\begin{array}{c}\text { Mets } \\
(N=209) \\
N(\%)\end{array}$ & $\begin{array}{c}\text { NO } \\
(N=505) \\
N(\%)\end{array}$ & $x^{2}$ & $\begin{array}{l}\text { Linear } \\
\text { Trend Test }\end{array}$ & $\begin{array}{c}\text { Mets } \\
(N=402) \\
N(\%)\end{array}$ & $\begin{array}{c}\text { NO } \\
(N=714) \\
N(\%)\end{array}$ & $x^{2}$ & $\begin{array}{c}\text { Linear } \\
\text { Trend Test }\end{array}$ \\
\hline \multicolumn{10}{|l|}{ Sociodemographic characteristics } \\
\hline Age & $\begin{array}{l}<50 \\
50-64 \\
\geq 65\end{array}$ & $\begin{array}{l}68(32.5) \\
66(31.6) \\
75(35.9)\end{array}$ & $\begin{array}{l}238(47.1) \\
121(24.0) \\
146(28.9)\end{array}$ & $12.944^{* *}$ & $9.485^{* *}$ & $\begin{array}{c}16(4.0) \\
80(19.9) \\
306(76.1)\end{array}$ & $\begin{array}{l}202(28.3) \\
151(21.1) \\
361(50.6)\end{array}$ & $106.124^{* * *}$ & $101.258^{* * *}$ \\
\hline BMI, $\mathrm{kg} / \mathrm{m}^{2}$ & $\begin{array}{l}<18.5 \\
18.5 \sim 24.9 \\
\geq 25\end{array}$ & $\begin{array}{c}2(1.0) \\
62(29.7) \\
145(69.4)\end{array}$ & $\begin{array}{c}26(5.1) \\
347(68.7) \\
132(26.1)\end{array}$ & $117.208^{* * *}$ & $108.685^{* * *}$ & $\begin{array}{c}1(0.2) \\
120(29.9) \\
281(26.9)\end{array}$ & $\begin{array}{c}42(5.9) \\
471(66.0) \\
201(28.2)\end{array}$ & $188.327^{* * *}$ & $182.747^{* * *}$ \\
\hline Subjective health status & $\begin{array}{l}\text { Good } \\
\text { normal } \\
\text { Poor }\end{array}$ & $\begin{array}{c}36(17.2) \\
109(52.2) \\
64(30.6)\end{array}$ & $\begin{array}{l}154(30.5) \\
247(48.9) \\
104(20.6)\end{array}$ & $16.411^{* * *}$ & $16.011^{* * *}$ & $\begin{array}{c}36(9.0) \\
190(47.3) \\
176(43.8)\end{array}$ & $\begin{array}{l}133(18.6) \\
360(50.4) \\
221(31.0)\end{array}$ & $28.307^{* * *}$ & $27.952 * * *$ \\
\hline Subjective body shape recognition & $\begin{array}{l}\text { Thin } \\
\text { normal } \\
\text { Fat }\end{array}$ & $\begin{array}{c}13(6.2) \\
78(37.3) \\
118(56.5)\end{array}$ & $\begin{array}{l}148(29.3) \\
232(45.9) \\
125(24.8)\end{array}$ & $81.137^{* * *}$ & $80.196^{* * *}$ & $\begin{array}{c}38(9.5) \\
133(33.1) \\
231(57.5)\end{array}$ & $\begin{array}{l}143(20.0) \\
311(43.6) \\
260(36.4)\end{array}$ & $50.724^{* * *}$ & $48.948^{* * *}$ \\
\hline Level of education & $\begin{array}{l}\text { Graduated from a college or higher } \\
\text { Graduated from a high school or less }\end{array}$ & $\begin{array}{c}65(31.1) \\
144(68.9)\end{array}$ & $\begin{array}{l}184(36.4) \\
321(63.6)\end{array}$ & 1.853 & 1.850 & $\begin{array}{c}25(6.2) \\
377(93.8)\end{array}$ & $\begin{array}{l}152(21.3) \\
562(78.7)\end{array}$ & $43.768^{* * *}$ & $43.728^{* * *}$ \\
\hline Household income & $\begin{array}{l}\text { Low } \\
\text { Mid-low } \\
\text { Mid-high } \\
\text { High }\end{array}$ & $\begin{array}{l}93(44.5) \\
47(22.5) \\
24(11.5) \\
45(21.5)\end{array}$ & $\begin{array}{c}181(35.8) \\
120(23.8) \\
105(20.8) \\
99(19.6)\end{array}$ & $10.354^{*}$ & 2.208 & $\begin{array}{c}290(72.1) \\
78(19.4) \\
23(5.7) \\
11(2.7)\end{array}$ & $\begin{array}{c}370(51.8) \\
173(24.2) \\
103(14.4) \\
68(9.5)\end{array}$ & $54.616^{* * *}$ & $53.354^{* * *}$ \\
\hline Employment & $\begin{array}{l}\text { Yes } \\
\text { No }\end{array}$ & $\begin{array}{l}122(58.4) \\
87(41.6)\end{array}$ & $\begin{array}{l}310(61.4) \\
195(38.6)\end{array}$ & 0.562 & & $\begin{array}{l}129(32.1) \\
273(67.9)\end{array}$ & $\begin{array}{l}361(50.6) \\
353(49.4)\end{array}$ & $35.627^{* * *}$ & \\
\hline Food security & $\begin{array}{l}\text { Enough } \\
\text { Not enough }\end{array}$ & $\begin{array}{l}179(85.6) \\
30(14.4)\end{array}$ & $\begin{array}{c}465(92.1) \\
40(7.9)\end{array}$ & $6.919^{* *}$ & $6.909^{* *}$ & $\begin{aligned} & 366(91.0) \\
& 36(9.0)\end{aligned}$ & $\begin{aligned} 668 & (93.6) \\
46 & (6.4)\end{aligned}$ & 2.385 & 2.383 \\
\hline \multicolumn{10}{|l|}{ Health-related characteristics } \\
\hline Alcohol intake & $\begin{array}{l}\text { No } \\
\text { Yes }\end{array}$ & $\begin{array}{c}85(40.7) \\
124(59.3)\end{array}$ & $\begin{array}{l}187(37.0) \\
318(63.0)\end{array}$ & .831 & & $\begin{array}{c}337(83.8) \\
65(16.2)\end{array}$ & $\begin{array}{l}512(71.7) \\
202(28.3)\end{array}$ & $20.765^{* * *}$ & \\
\hline Smoking status & $\begin{array}{l}\text { No } \\
\text { Yes }\end{array}$ & $\begin{array}{c}43(20.6) \\
166(79.4)\end{array}$ & $\begin{array}{l}123(24.4) \\
382(75.6)\end{array}$ & 1.185 & & $\begin{array}{c}356(88.6) \\
46(11.4)\end{array}$ & $\begin{array}{l}620(86.8) \\
94(13.2)\end{array}$ & 0.696 & \\
\hline Exercise & $\begin{array}{l}\text { No } \\
\text { Yes }\end{array}$ & $\begin{array}{c}164(78.5) \\
45(21.5)\end{array}$ & $\begin{array}{l}349(69.1) \\
156(30.9)\end{array}$ & $6.403 *$ & & $\begin{array}{c}371(92.3) \\
31(7.7)\end{array}$ & $\begin{array}{l}582(81.5) \\
132(18.5)\end{array}$ & $23.945^{* * *}$ & \\
\hline Sleep hours & $\begin{array}{l}\geq 8 \\
<8\end{array}$ & $\begin{array}{c}135(64.6) \\
74(35.4)\end{array}$ & $\begin{array}{l}375(74.3) \\
130(25.7)\end{array}$ & $6.765^{* *}$ & $6.755^{* *}$ & $\begin{array}{l}262(65.2) \\
140(34.8)\end{array}$ & $\begin{array}{l}497(69.6) \\
217(30.4)\end{array}$ & 2.324 & 2.322 \\
\hline
\end{tabular}


Table 3. Odds ratios and 95\% confidence intervals for Mets in the multiple logistic regression analysis $(N=1830)$.

\begin{tabular}{|c|c|c|c|c|c|}
\hline \multirow[t]{2}{*}{ Variable } & \multirow[t]{2}{*}{ Categories } & \multicolumn{2}{|r|}{ Men } & \multicolumn{2}{|r|}{ Women } \\
\hline & & OR & $95 \%$ CI & OR & $95 \% \mathrm{CI}$ \\
\hline \multicolumn{6}{|l|}{ Sociodemographic characteristics } \\
\hline \multirow[t]{3}{*}{ Age } & $<50$ & 1 & & 1 & \\
\hline & $50-64$ & 1.996 & $1.199-3.322 * *$ & 5.568 & $2.924-10.606^{* * *}$ \\
\hline & $\geq 65$ & 1.846 & $1.063-3.204$ * & 6.001 & $3.088-11.661 * * *$ \\
\hline \multirow[t]{3}{*}{ BMI, $\mathrm{kg} / \mathrm{m}^{2}$} & $<18.5$ & 1 & & 1 & \\
\hline & $18.5 \sim 24.9$ & 1.759 & $.371-8.343$ & 8.176 & $1.077-62.045 *$ \\
\hline & $\geq 25$ & 7.963 & $1.587-39.949 *$ & 34.636 & $4.461-268.934^{* *}$ \\
\hline \multirow[t]{3}{*}{ Subjective health status } & Good & 1 & & 1 & \\
\hline & normal & 1.805 & $1.106-2.946 *$ & 1.562 & $0.979-2.491$ \\
\hline & Poor & 2.070 & $1.169-3.667^{*}$ & 1.819 & $1.111-2.980 *$ \\
\hline \multirow[t]{3}{*}{ Subjective body shape recognition } & Thin & 1 & & 1 & \\
\hline & normal & 2.930 & $1.466-5.857^{* *}$ & 1.345 & $0.836-2.163$ \\
\hline & Fat & 4.674 & $2.082-10.495^{* * * *}$ & 1.627 & $0.964-2.746$ \\
\hline \multirow[t]{2}{*}{ Level of education } & $\begin{array}{l}\text { Graduated from a } \\
\text { college or higher }\end{array}$ & 1 & & 1 & \\
\hline & $\begin{array}{l}\text { Graduated from a high } \\
\text { school or less }\end{array}$ & 1.114 & 0.698-1.777 & 1.124 & $0.632-2.000$ \\
\hline \multirow[t]{4}{*}{ Household income } & Low & 1 & & 1 & \\
\hline & Mid-low & 0.613 & $0.351-1.069$ & 0.901 & $0.614-1.322$ \\
\hline & Mid-high & 0.429 & $0.217-0.850$ * & 1.026 & $0.554-1.898$ \\
\hline & High & 0.889 & $0.457-1.730$ & 0.738 & $0.335-1.625$ \\
\hline \multirow[t]{2}{*}{ Employment } & Yes & 1 & & 1 & \\
\hline & No & 0.853 & $0.519-1.401$ & 1.170 & $0.843-1.624$ \\
\hline \multirow[t]{2}{*}{ Food security } & Enough & 1 & & 1 & \\
\hline & Not Enough & 1.608 & $0.848-3.048$ & 1.056 & $0.624-1.789$ \\
\hline \multicolumn{6}{|l|}{ Health-related characteristics } \\
\hline \multirow[t]{2}{*}{ Alcohol intake } & No & 1 & & 1 & \\
\hline & Yes & 0.934 & $0.631-1.384$ & 0.725 & $0.496-1.059$ \\
\hline \multirow[t]{2}{*}{ Exercise } & No & 1 & & 1 & \\
\hline & Yes & 0.653 & $0.409-1.040$ & 0.568 & $0.350-0.9210 *$ \\
\hline \multirow[t]{2}{*}{ Sleep hours } & $\geq 8$ & 1 & & 1 & \\
\hline & $<8$ & 1.782 & $1.180-2.690$ ** & 1.392 & $1.024-1.8930$ * \\
\hline
\end{tabular}

${ }^{*} p<0.05,{ }^{* *} p<0.01,{ }^{* * *} p<0.0001$.

\section{Discussion}

In this study, we analyzed the effects of the socioeconomic status and health behaviors of single-person households on the risk of developing Mets according to sex. The results of this study showed that the factors that influence the development of Mets in men from single-person households are age, BMI, subjective health status, subjective recognition of body shape, household income, and sleep hours, whereas for women from single-person households, the factors are age, BMI, subjective health status, exercise, and sleep hours. Of the 888 persons from single-person households aged 65 years or older, $221(24.9 \%)$ were men and $667(75.1 \%)$ were women. This finding is similar to the results of a previous study [22]. We also found that, even within the same single-person household, there may be differences in the health problems that individuals experience due to different age distributions. In a preliminary study on the health problems of single-person households based on generation, drinking and smoking were high among the young and chronic diseases were high among the elderly [23]. In the present study, the distribution of 888 single-person households over the age of 65 was differentiated by sex. In this regard, the study results also showed that 
men had higher rates of drinking and smoking than women, while the incidence of chronic disease-causing Mets was higher in women.

Men subjectively evaluated their health status and perceived body type more positively than women in this study. Among men, 76.5\% rated their health status as above normal, whereas among women, $84.9 \%$ rated their health status as below normal. This finding is also similar to the results of previous studies in which men reported better subjective health statuses than women [24]. It has been reported that subjective health status is affected by socioeconomic status [24]. In the present study, a higher proportion of women (59.1\%) than men (38.4\%) reported a low household income. In addition, most of the men were employed $(60.5 \%)$, whereas most of the women were unemployed (56.1\%). These results suggest that socioeconomic level may influence an individual's subjective perception of health. More women $(44.0 \%)$ considered their subjective body shape recognition as "fat" than men (34.0\%). Among the subjects who recognized their body shape as "fat" in the normal to overweight group with a BMI of 18.5-24.9, 38 out of 243 men $(15.6 \%)$ and 135 out of 491 women $(27.5 \%)$ showed a significant difference $(p<0.0001)$. In previous studies, more women thought they were obese even though their body shape was normal compared to men $[25,26]$. In this study, the reason for this trend is that although there was no significant difference in the distribution of BMI between men and women $(p=0.172)$, there was a significant difference according to sex in subjective body shape recognition $(p<0.0001)$. In men, the group that subjectively perceived their body type as normal or obese had a higher risk of Mets than those who subjectively perceived their body shape as thin. In women, there was no significant difference in the prevalence of Mets according to the subjective recognition of body shape. This may also be because women tend to have a distorted view of their body shape compared to their actual weight.

Age, BMI, subjective health status, and sleep hours were identified as significant variables that influence the development of Mets in both men and women. This is consistent with previous study results where the older the age [9], the higher the BMI [27], and the poorer the subjective health status [28], the higher the incidence of Mets. In the present study, the incidence of Mets was significantly higher in those who slept for less than $8 \mathrm{~h}$ than in those who slept for $8 \mathrm{~h}$ or longer. Existing studies on the relationship between sleep hours and the onset of Mets have shown that extremely short sleep hours or long sleep hours increase the risk of developing Mets [29,30]. This indicates that sleeping less or sleeping for longer than appropriate increases the risk of Mets. Therefore, in future studies, it is necessary to analyze sleep hours by stratifying or grouping them.

The present study results showed that income influences the prevalence of Mets in men, whereas exercise affects the prevalence of Mets in women. Previous studies that analyzed the KNHANES data showed different results regarding the relationship between exercise and Mets because the definition of exercise in each study varied [31]. In those studies, exercise was evaluated as per the subject's activity level, which was reported in a questionnaire. In the present study, exercise was evaluated as the level of physical activity. A more objective method that can be used to evaluate the amount and duration of exercise is needed. A previous study in which the relationship between household income and Mets was investigated according to sex showed a significant relationship between household income and Mets in women [32]. However, no significant relationship was observed in the present study. The findings of that previous study indicated that the relationship between income and Mets is insignificant at 65 years or older, even in women. Most of the women in the present study were 65 years or older (59.8\%) [32]. Thus, the absence of a significant relationship between household income and Mets in women in the present study can be attributed to the characteristics of the subject group.

This study has several limitations. First, although the stratified clustering sampling method used to extract the samples from the KNHANES ensured that the data were reliable, the method may not have sufficiently represented the increasing number of single-person households each year; hence, a better data extraction method is needed. Second, we used the self-report method to measure alcohol intake, exercise, and sleep hours. This method 
has limitations in measuring an objective quantity. However, despite these limitations, this study is significant in that it is easy to generalize and apply the results when the KNHANES data are used. Second, this study identified major health problems that existed at different levels, even in the same single-person household, due to differences in sex and age distribution. Third, this study demonstrated the need to consider the differences in health behaviors according to sex and age when preparing health promotion measures for reducing the prevalence of Mets in single-person households.

\section{Conclusions}

In this study, we analyzed the factors that affect the development of Mets in singleperson households according to sex. The results showed that men from single-person households have higher rates of drinking and smoking, which are negative health behaviors, and a higher rate of exercise, which is a positive health behavior, than women. The results also showed that the prevalence of Mets was higher among women than among men from single-person households. A multiple logistic regression analysis showed that significant risk factors for Mets in men were age, BMI, subjective health status, subjective recognition of body shape, household income, and sleep hours, whereas for women, age, BMI, subjective health status, exercise, sleep hours were risk factors. Based on the results of this study, we recommend the differentiated management of health interventions for single-person households. Further, it is necessary to prepare educational interventions or policies aimed at reducing the risk factors for Mets identified in the present study for men and women in single-person households.

Author Contributions: Conceptualization, J.K. and H.C.; methodology, J.K.; writing-original draft preparation, J.K. and H.C.; writing-review and editing, J.K. and H.C.; visualization, J.K.; supervision, H.C. All authors have read and agreed to the published version of the manuscript.

Funding: This research received no external funding.

Institutional Review Board Statement: The study was conducted according to the guidelines of the Declaration of Helsinki, and approved by the Institutional Review Board of Korea Centers for Disease Control and Prevention (2018-01-03-P-A).

Informed Consent Statement: Informed consent was obtained from all subjects involved in the study.

Data Availability Statement: Data are available from the Korea National Health and Nutrition Examination Survey (KNHANES), conducted by the Korea Centers for Disease Control and Prevention (KCDCP), and are freely available from KCDCP (https:/ / knhanes.kdca.go.kr).

Conflicts of Interest: The authors declare no conflict of interest.

Ethics Statement: All participants voluntarily agreed to participate in the study prior to the start of the survey by completing an informed consent form. The Korea National Health and Nutrition Examination Survey was approved by the Institutional Review Board of Korea Centers for Disease Control and Prevention (2018-01-03-P-A).

\section{References}

1. Jung, S.H. The Latest Trends of Single-Person Households Related Policy in U.S.A. Glob. Soc. Secur. Rev. 2019, 11, 37-49. Available online: http:/ / repository.kihasa.re.kr/bitstream/201002/33523/1/\%28\%EA\%B2\%A8\%EC\%9A\%B8\%ED\%98\%B8\%29_\%EA\% B8\%B0\%ED\%9A\%8D4_\%EC\%A0\%95\%EC\%88\%9C\%ED\%9D\%AC.pdf (accessed on 20 March 2021).

2. Eurostat. Number of Private Households by Household Composition, Number of Children and Age of Youngest Child (1000). 2019. Available online: https://ec.europa.eu/eurostat/databrowser/view/LFST_HHNHTYCH_custom_518387/default/table? lang=en (accessed on 20 March 2021).

3. U.S. Census Bureau. Census. 2019. Available online: https://www.census.gov (accessed on 20 March 2021).

4. Statistics Japan. Japan Statistical Yearbook. 2019. Available online: https://www.stat.go.jp/data/nenkan/68nenkan/zenbun/jp6 8/index_h5.html\#66 (accessed on 20 March 2021).

5. Statistics Korea. 2020 Population and Housing Census. Available online: https://census.go.kr/mainView.do\#infographic (accessed on 21 March 2021). 
6. Statistics Korea. Household Projections by Province 2017 2047. 2019. Available online: http://kostat.go.kr/portal/korea/kor_ $\mathrm{nw} / 1 / 2 / 6 /$ index.board? bmode $=$ read\&bSeq=\&aSeq $=377540 \&$ pageNo=1\&rowNum=10\&navCount=10\&currPg=\&searchInfo= \&sTarget=title\&sTxt $=$ (accessed on 20 March 2021).

7. Gu, H.J. Analysis of health-related quality of life factors in south Korean one-person households. J. Digit. Converg. 2019, $17,453-463$.

8. Cho, Y.K.; Shim, K.W.; Suk, H.W.; Lee, H.S.; Lee, S.W.; Byun, A.R.; Lee, H.N. Differences between one-person and multi-person households on socioeconomic status, health behavior, and metabolic syndrome across gender and age groups. Korean J. Fam. Pract. 2019, 9, 373-382. [CrossRef]

9. An, B.; Son, J. Analysis of metabolic syndrome in Korean adult one-person households. J. Korean Public Health Nurs. 2018, $31,30-43$.

10. Alberti, K.G.; Eckel, R.H.; Grundy, S.M.; Zimmet, P.Z.; Cleeman, J.I.; Donato, K.A.; Fruchart, J.C.; James, W.P.; Loria, C.M.; Smith, S.C., Jr.; et al. Harmonizing the metabolic syndrome: A joint interim statement of the International Diabetes Federation Task Force on Epidemiology and Prevention; National Heart, Lung, and Blood Institute; American Heart Association; World Heart Federation; International Atherosclerosis Society; and International Association for the Study of Obesity. Circulation 2009, 120, 1640-1645.

11. Kim, Y.J. Association of Family Composition and Metabolic Syndrome in Korean Adults Aged over 45 Years Old. Asian Nurs. Res. 2015, 9, 349-355. [CrossRef] [PubMed]

12. Aguilar, M.; Bhuket, T.; Torres, S.; Liu, B.; Wong, R.J. Prevalence of the metabolic syndrome in the United States, 2003-2012. JAMA 2015, 313, 1973-1974. [CrossRef] [PubMed]

13. Li, Y.; Zhao, L.; Yu, D.; Wang, Z.; Ding, G. Metabolic syndrome prevalence and its risk factors among adults in China: A nationally representative cross-sectional study. PLoS ONE 2018, 13, e0199293. [CrossRef] [PubMed]

14. Kim, M.H.; Lee, S.H.; Shin, K.S.; Son, D.Y.; Kim, S.H.; Joe, H.; Yoo, B.W.; Hong, S.H.; Cho, C.Y.; Shin, H.S.; et al. The change of metabolic syndrome prevalence and its risk factors in Korean adults for decade: Korea National Health and Nutrition Examination Survey for 2008-2017. Korean J. Fam. Pract. 2020, 10, 44-52. [CrossRef]

15. Seo, J.M.; Lim, N.K.; Lim, J.Y.; Park, H.Y. Gender difference in association with socioeconomic status and incidence of metabolic syndrome in Korean adults. Korean J. Obes. 2016, 25, 247-254. [CrossRef]

16. Xi, B.; He, D.; Hu, Y.; Zhou, D. Prevalence of metabolic syndrome and its influencing factors among the Chinese adults: The China Health and Nutrition Survey in 2009. Prev. Med. 2013, 57, 867-871. [CrossRef]

17. Moore, J.X.; Chaudhary, N.; Akinyemiju, T. Metabolic Syndrome Prevalence by Race/Ethnicity and Sex in the United States, National Health and Nutrition Examination Survey, 1988-2012. Prev. Chronic Dis. 2017, 14, E24. [CrossRef] [PubMed]

18. Pucci, G.; Alcidi, R.; Tap, L.; Battista, F.; Mattace-Raso, F.; Schillaci, G. Sex- and gender-related prevalence, cardiovascular risk and therapeutic approach in metabolic syndrome: A review of the literature. Pharmacol. Res. 2017, 120, 34-42. [CrossRef] [PubMed]

19. Seo, M.H.; Lee, W.Y.; Kim, S.S.; Kang, J.H.; Kang, J.H.; Kim, K.K.; Kim, B.Y.; Kim, Y.H.; Kim, W.J.; Kim, E.M.; et al. 2018 Korean Society for the Study of Obesity Guideline for the Management of Obesity in Korea. J. Obes. Metab. Syndr. 2019, $28,40-45$. [CrossRef]

20. World Health Organization. Measuring Obesity: Classification and Description of Anthropometric Data: Report on a WHO Consultation on the Epidemiology of Obesity, Warsaw, 21-23 October 1987; World Health Organization, Regional Office for Europe: Copenhagen, Denmark, 1989; p. 123.

21. Executive Summary of The Third Report of The National Cholesterol Education Program (NCEP) Expert Panel on Detection, Evaluation, And Treatment of High Blood Cholesterol In Adults (Adult Treatment Panel III). JAMA 2001, 285, $2486-2497$. [CrossRef]

22. Song, Y. Current Conditions of Single-Household Female Elderly and Improvement Measures at Policy Level. Ewha J. Gender Law 2015, 7, 33-72.

23. Kang, E.; Lee, M. Single-Person Households in South Korea and Their Policy Implications. Health and Welfare Policy Forum; Korea Institute for Health and Social Affairs: Sejong, Korea, 2016; pp. 47-56.

24. Lee, J.M.; Kim, W.J.; Sohn, H.S.; Chun, H.J.; Lee, M.J.; Park, H.S. Influences on Health Behaviors Execution and Self Rated Health as Socioeconomic Class by the Age Bracket. J. Korea Contents Assoc. 2012, 12, 317-327. [CrossRef]

25. Lee, H.; Chung, W.; Lim, S.; Han, E. Association of a Combination between Actual Body Mass Index Status and Perceived Body Image with Anxiety and Depressive Condition in Korean Men and Women: The Fifth and Sixth Korea National Health and Nutrition Examination Survey (2010-2014). Health Policy Manag. 2018, 28, 3-14.

26. Craig, P.L.; Caterson, I.D. Weight and perceptions of body image in women and men in a Sydney sample. Community Health Stud. 1990, 14, 373-383. [CrossRef]

27. Park, H.R.; Cho, Y.C. Prevalence Rates of Risk Factors of Metabolic Syndrome, and Its Related with Obesity Indices among the Health Checkup Examinees. J. Korea Acad. Ind. Coop. Soc. 2007, 8, 173-178.

28. Botoseneanu, A.; Ambrosius, W.T.; Beavers, D.P.; de Rekeneire, N.; Anton, S.; Church, T.; Folta, S.C.; Goodpaster, B.H.; King, A.C.; Nicklas, B.J.; et al. Prevalence of metabolic syndrome and its association with physical capacity, disability, and self-rated health in Lifestyle Interventions and Independence for Elders Study participants. J. Am. Geriatr. Soc. 2015, 63, 222-232. [CrossRef]

29. Smiley, A.; King, D.; Bidulescu, A. The Association between Sleep Duration and Metabolic Syndrome: The NHANES $2013 / 2014$. Nutrients 2019, 11, 2582. [CrossRef] [PubMed] 
30. Stefani, K.M.; Kim, H.C.; Kim, J.; Oh, K.; Suh, I. The influence of sex and age on the relationship between sleep duration and metabolic syndrome in Korean adults. Diabetes Res. Clin. Pract. 2013, 102, 250-259. [CrossRef] [PubMed]

31. Im, M.Y.; Lee, Y.R.; Han, S.J.; Cho, C.M. The Effects of Lifestyle Factors on Metabolic Syndrome among Korean Adults. J. Korean Acad Community Health Nurs. 2012, 23, 13-21. [CrossRef]

32. Loucks, E.B.; Magnusson, K.T.; Cook, S.; Rehkopf, D.H.; Ford, E.S.; Lisa, F.B. Socioeconomic Position and the Metabolic Syndrome in Early, Middle, and Late Life: Evidence from NHANES 1999-2002. Ann. Epidemiol. 2007, 17, 782-790. [CrossRef] [PubMed] 